\title{
Thermodynamic instabilities in hot and dense nuclear matter
}

\author{
A. Lavagno ${ }^{1,2, \star}$ \\ ${ }^{1}$ Department of Applied Science and Technology, Politecnico di Torino, Torino, Italy \\ ${ }^{2}$ Istituto Nazionale di Fisica Nucleare (INFN), Sezione di Torino, Torino, Italy
}

\begin{abstract}
We study the presence of thermodynamic instabilities in a hot and dense nuclear medium where a nuclear phase transition can take place. Similarly to the low density nuclear liquid-gas phase transition, we show that such a phase transition is characterized by pure hadronic matter with both mechanical instability (fluctuations on the baryon density) that by chemical-diffusive instability (fluctuations on the strangeness concentration). The analysis is performed by requiring the global conservation of baryon number and zero net strangeness in the framework of an effective relativistic mean field theory with the inclusion of the $\Delta(1232)$-isobars, hyperons and the lightest pseudoscalar and vector meson degrees of freedom. It turns out that in this situation hadronic phases with different values of strangeness content may coexist, altering significantly meson-antimeson ratios.
\end{abstract}

We investigate the nuclear medium in the context of relativistic mean field approach, where the nuclear force is mediated by the exchange of isoscalar-scalar $(\sigma)$, isoscalar-vector $(\omega)$ and isovectorvector $(\rho)$ mesons fields in the so-called TM1 parameter set [1, 2]. Hyperon degrees of freedom are included taking into account of the determination of the corresponding meson-hyperon coupling constants that have been fitted to hypernuclear properties [3, 4].

In regime of finite values of temperature and density, a state of high density resonance matter may be formed and the $\Delta(1232)$-isobar degrees of freedom are expected to play a central role [5-8]. In particular, the formation of resonances matter contributes essentially to baryon stopping, hadronic flow effects and enhanced strangeness. Following Ref.s [2, 7], we take into account of the $\Delta$-isobar degree of freedoms.

We are dealing with the study of a multi-component system at finite temperature and density with two conserved charges: baryon (B) number and zero net strangeness (S) number $\left(r_{S}=\rho_{S} / \rho_{B}=0\right)$. For what concern the electric charge $(\mathrm{Q})$, we work in symmetric nuclear matter with a fixed value of $Z / A=0.5$ and we do not consider fluctuations in the electric charge fraction, due to the high temperature regime considered in the present investigation. Therefore, the electric charge results to be separately conserved in each phase during the phase transition and the chemical potential of particle of index $i$ can be written as $\mu_{i}=b_{i} \mu_{B}+s_{i} \mu_{S}$, where $b_{i}$ and $s_{i}$ are, respectively, the baryon and the strangeness quantum numbers of $i$-th hadronic species.

By increasing the temperature and the baryon density during the high energy heavy ion collisions, a multi-particle system may take place and the formation of antiparticles become much more relevant. In analogy with the liquid-gas case [9], we are going to investigate the existence of a possible phase transition in the nuclear medium by studying the presence of instabilities (mechanical and/or chemical) in the nuclear equation of state.

^e-mail: andrea.lavagno@polito.it 
For such a system, the Helmholtz free energy density $F$ can be written as

$$
F\left(T, \rho_{B}, \rho_{S}\right)=-P\left(T, \mu_{B}, \mu_{S}\right)+\mu_{B} \rho_{B}+\mu_{S} \rho_{S},
$$

with

$$
\mu_{B}=\left(\frac{\partial F}{\partial \rho_{B}}\right)_{T, \rho_{S}}, \quad \mu_{S}=\left(\frac{\partial F}{\partial \rho_{S}}\right)_{T, \rho_{B}} .
$$

In a system with $N$ different particles, the particle chemical potentials are expressed as the linear combination of the two independent chemical potentials $\mu_{B}$ and $\mu_{S}$ and, as a consequence, $\sum_{i=1}^{N} \mu_{i} \rho_{i}=$ $\mu_{B} \rho_{B}+\mu_{S} \rho_{S}$.

Assuming the presence of two phases (denoted as $I$ and $I I$, respectively), the system is stable against the separation in two phases if the free energy of a single phase is lower than the free energy in all two phases configuration. The phase coexistence is given by the Gibbs conditions

$$
\begin{aligned}
& \mu_{B}^{I}=\mu_{B}^{I I}, \quad \mu_{S}^{I}=\mu_{S}^{I I}, \\
& P^{I}\left(T, \mu_{B}, \mu_{S}\right)=P^{I I}\left(T, \mu_{B}, \mu_{S}\right) .
\end{aligned}
$$

At a given baryon density $\rho_{B}$ and at a given zero net strangeness density $r_{S}=\rho_{S} / \rho_{B}=0$, the chemical potentials $\mu_{B}$ are $\mu_{S}$ are univocally determined by the following equations

$$
\begin{aligned}
& \rho_{B}=(1-\chi) \rho_{B}^{I}\left(T, \mu_{B}, \mu_{S}\right)+\chi \rho_{B}^{I I}\left(T, \mu_{B}, \mu_{S}\right), \\
& \rho_{S}=(1-\chi) \rho_{S}^{I}\left(T, \mu_{B}, \mu_{S}\right)+\chi \rho_{S}^{I I}\left(T, \mu_{B}, \mu_{S}\right),
\end{aligned}
$$

where $\rho_{B}^{I(I I)}$ and $\rho_{S}^{I(I I)}$ are, respectively, the baryon and strangeness densities in the low density (I) and in the higher density $(I I)$ phase and $\chi$ is the volume fraction of the phase $I I$ in the mixed phase $(0 \leq \chi \leq 1)$. An important feature of this conditions is that, unlike the case of a single conserved charge, baryon and strangeness densities can be different in the two phases, although the total $\rho_{B}$ and $\rho_{S}$ are fixed.

For a system in thermal equilibrium, the possible phase transition can be characterized by mechanical (fluctuations in the baryon density) and chemical instabilities (fluctuations in the strangeness number). The mechanical and chemical stability conditions $\left(r_{S}=\rho_{S} / \rho_{B}\right)$ are satisfied if [7]:

$$
\rho_{B}\left(\frac{\partial P}{\partial \rho_{B}}\right)_{T, \rho_{C}}>0, \quad\left(\frac{\partial \mu_{S}}{\partial r_{S}}\right)_{T, P}>0 .
$$

In Fig. 1, we show the pressure as a function of baryon density at fixed temperature. The continuous (dashed) lines correspond to the solution obtained with (without) the Gibbs construction. In this case the conditions of mechanical and chemical stability are not satisfied and the system goes to a phase transition. Let us observe that, although the system has globally zero net strangeness $\left(r_{S}=0\right)$, the mixed phase is realized with two phase with different and finite values of strangeness and different baryon-antibaryon content (baryon and strangeness charges are globally conserved but are different in the two hadronic phases).

The above property can imply remarkably consequences in the meson-antimeson production and in the strangeness content at different temperatures and baryon densities. This matter of fact can be observed, for example, in Fig. 2, where the $K^{+} / K^{-}$ratios, as a function of the baryon density, are reported for different temperatures $\left(x_{\sigma \Delta}=g_{\sigma \Delta} / g_{\sigma N}=1.2\right.$ is the used $\sigma$ meson- $\Delta$ ratio coupling constant and $\left.x_{\omega \Delta}=g_{\omega \Delta} / g_{\omega N}=1\right)$. The black points in the isothermal curves stand for the beginning and the end of the phase transition. We observe that at lower $(T \leq 100 \mathrm{MeV})$ and higher $(T \geq 150 \mathrm{MeV})$ temperatures, the phase transition does not occur, while, in the intermediate range of temperature 


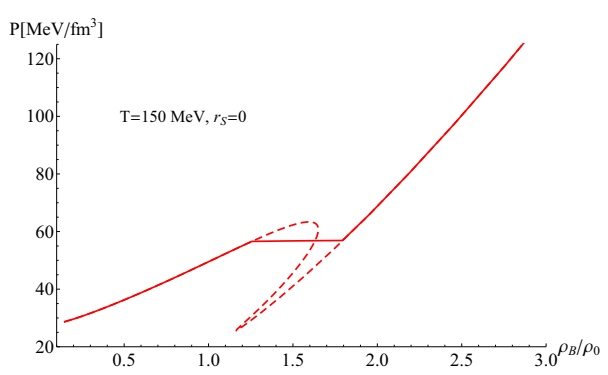

Figure 1. Pressure as a function of baryon density at fixed temperature. The continuous (dashed) lines correspond to the solution obtained with (without) the Gibbs construction.

$(100<T<150 \mathrm{MeV})$, thermodynamic instabilities take place and this feature can imply an abrupt variation of the ratio during the phase transition region. Such behavior could be phenomenological relevant in order to identify the presence of such phase transition in the future compressed baryonic matter experiments [10].

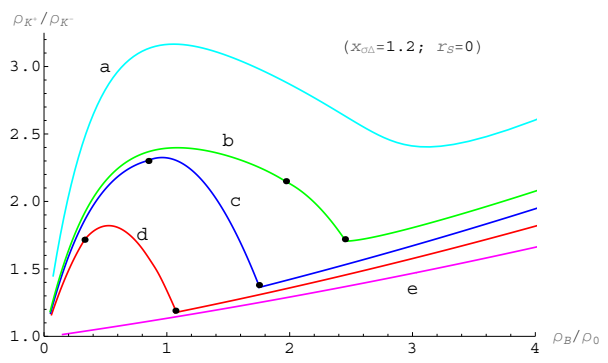

Figure 2. Kaon to antikaon ratios as a function of baryon density at different temperatures (labels $a$ to $e$ correspond to $T=120,127,135,140,150 \mathrm{MeV}$, respectively). The black points stand for the beginning and the end of the phase transition.

\section{References}

[1] N.K. Glendenning and S.A. Moszkowski, Phys. Rev. Lett. 672414 (1991)

[2] A. Lavagno, Phys. Rev. C 81044909 (2010)

[3] N.K. Glendenning and J. Schaffner-Bielich, Phys. Rev. C 60025803 (1999)

[4] A. Drago, A. Lavagno, G. Pagliara, Phys. Rev. D 89043014 (2014)

[5] M. Hofmann, R. Mattiello, H. Sorge, H. Stocker, and W. Greiner, Phys. Rev. C 51, 2095 (1995)

[6] Z. Li, G. Mao, Y. Zhuo, and W. Greiner, Phys. Rev. C 56, 1570 (1997)

[7] A. Lavagno, D. Pigato, Phys. Rev. C 86, 024917 (2012)

[8] A. Drago, A. Lavagno, G. Pagliara, D. Pigato, Phys. Rev. C 90, 065809 (2014)

[9] H. Müller, B.D. Serot, Phys. Rev. C 52, 2072 (1995)

[10] P. Senger, Nucl. Phys. A 862-863, 139 (2011) 